\title{
La teoría de redes sociales
}

\section{Carlos Lozares}

Universitat Autònoma de Barcelona. Departament de Sociologia. 08193 Bellaterra (Barcelona). Spain

\section{Resumen}

La teoría de redes sociales constituye un buen ejemplo de un tipo de aproximación en que teoría, aparato conceptual, métodos y técnicas de investigación han estado mutuamente sostenidos y generados. La perspectiva innovadora que aporta esta teoría es la relacional dentro de una vocación claramente estrucrural, es decir, aquella en que los vínculos o las relaciones entre entidades son unidades básicas de recogida de información y del análisis. En este attículo se hace una incroducción general a la reoria de redes; más adelante, en otros números đe la revista, se aportarán análisis empiricos.

Palabras clave: metodología, redes sociales, estructura social.

\section{Abstract. The theory of social networks}

The theory of Social Networks is an approach where theory, conceptual machinery, methods and research rechniques are reciprocally supported. The new perspective that this theory introduces in Sociology is the relational one with a structural orientation, that is to say, that one where the links or relationships between entities are the basic unites of gerting information and of doing analysis. This paper is a general introduction to Network Theory; in the future, we will contribute with empirical analysis.

Key words: methodology, social networks, social structure.

\section{Sumario}

\section{Origen y desarrollo}

2. Definición: que son las redes sociales

3. La perspecriva relacional de las redes sociales y la individualista-atributiva.

Datos, lenguajes y niveles de tratamiento

4. Las redes, un enfoque estructural
5. Perspectivas a modelos de tratamiento en las redes sociales, criterios (intuiciones) para analizar la estructura relacional y principios de análisis

G. Reflexiones finales

Bibliografia 


\section{Origen y desarrollo}

\section{Origenes e influencias en la teoria de redes}

La teoría de redes es deudora de diferentes corrientes de pensamiento y teorías: antropológica, psicológica, sociológica y también matemática. Estos orígenes históricos han sido excelentemente tratados por J. Scott (1991:1-38).

Scotr se remonta a la Gestait Theory para encontrar sus primeros orígenes. Dentro de esta concepción la percepción de un objeto se realiza dentro de una totalidad conceptual compleja y organizada, totalidad que posee propiedades específicas diferentes de la simple adición de las propiedades de las partes. La teoría de Kurr Lewin puso de relieve el hecho de que la percepción y el comportamiento de los individuos de un grupo, así como la misma estructura del grupo, se inscriben en un espacio social formado por dicho grupo y su entorno, configurando así un campo de relaciones. Estas relaciones pueden ser analizadas formalmente por procedimientos matemáticos.

En esta misma línea se inscribe también Moreno (1934) y su sociometría, quien puede ser considerado como uno de los fundadores de la teoría de redes bajo el nombre de sociometria. Moreno se interesó por la estrucrura de los grupos de amigos aunque fuera por razones terapéuticas. En la misma corriente teórica sitúa también Scott los grupos dinámicos de F. Heider (1946).

La teoría matemárica de grafos (Cartwright y Zander, 1953; Harary y Norman, 1953; Bavelas, 1948, 1950; Festinger, 1949) trata, por otro lado, de formalizar los estudios de Lewin, Moreno y Heider. Todos ellos, Moreno, Heider, Bavelas, Festinger, Cartwright (1959), Newcomb (1961) fueron psicólogos que trabajaron sobre los pequeños grupos a partir de los modelos de grafos teóricos para dar cuenta de la estructura social de los mismos y para poner de manifiesto cómo la estructura del grupo afecta a los comportamientos individuales (J. Galaskiewicz y S. Wasserman, 1993).

Otra de las fuentes u orígenes de las redes proviene del estructural-funcionalismo antropológico desarrollado en Harvard en los años treinta a cuarenta por L. Warner y E. Mayo a través de sus investigaciones sobre la estructura de los subgrupos en sus trabajos en la Hawthorne, factoría eléctrica de Chicago. Los análisis de los grupos y subgrupos a partir de la utilización de sociogramas jugó un papel importante. Warner trabajó también, entre los treinta y los treinta y cinco, en un estudio sobre la pequeña ciudad de Newburyport, Yankee City en N. England, sobre una base antropológica: observación, entrevistas y documentos históricos, y promovió los estudios de Old City.

Para Scott no hay constancia de la existencia de influencias mutuas entre la corriente que viene de la Gestalt y Grafos y la del estructural-funcionalismo antropológico entre 1930 y 1940 .

En la línea del estructural-funcionalismo, aunque con diferencias, aparece la escuela antropológica de Manchester, con Max Gluckman como figura importante. Sus rasgos característicos se pueden resumir en dos aspectos: primero, en la insistencia en el conflicto más que en la cohesión como factor del mantenimiento y la transformación del grupo y, segundo, y en consecuencia 
la visión de la estructura como redes relacionales analizables por técnicas específicas y como concepros sociológicos basados en la teoría del conflicto.

Como punto de confluencia entre el estructural-funcionalismo antropológico a través de Max Gluckman y los grupos dinámicos de F. Heider aparecen Barnes, Bott y Nadel.

J.A. Barnes (1954) analiza la importancia de las relaciones informales e interpersonales como la amistad, el parentesco y la vecindad en la integración de una pequeña comunidad de pescadores. La totalidad de la vida social se ha de contemplar "como un conjunto de algunos puntos (nodos) que se vinculan por líneas para formar redes totales de relaciones. La esfera informal de relaciones interpersonales se contempla así como una parte, una red parcial de una total" (J.A. Barnes, 1954:43 referenciado en Scott). Bott estudió la vida de familias británicas, sobre todo sus relaciones de parentesco a través del diseno de redes ( $\mathrm{E}$. Bott, 1955, 1956). Nadel parte de la definición de estructura como la articulación de elementos en la formación de una totalidad (S.F. Nadel, 1957). Las estructuras sociales son estructuras de roles. Separando su contenido de la forma Nadel piensa que la estructura puede ser investigada por métodos comparativos y por modelos formales matemáticos.

Las redes sociales tienen pues una rica tradición en la antropología cultural. Hacia mediados de los cincuenta los antropólogos estudian la urbanización, particularmente J.A. Barnes (1954), y encuentran que las aproximaciones de la tradición antropológica a las organizaciones sociales no son suficientemente ricas en las sociedades complejas. Se necesitan pues nuevos conceptos para cuantificar las relaciones en el trabajo de campo, conceptos como apertura, conectividad, círculo social, densidad, etc. aplicables a las siruaciones sociales estudiadas.

J.C. Mitchell (1969) es posiblemente quien mejor representa la convergencia de ambas escuelas, la que viene del estructural-funcionalismo antropológico a través de Warner, Gluckman hasta Barnes, Bott y Nadel y la que proviene de la teoría de grafos, Köening, Cartwright, Zander, Harary, Norman y Bavelas poniendo las bases sistemáticas para el análisis social de las redes.

Los cincuenta y sesenta no son de excesiva dedicación de los sociólogos a las redes sociales. No obstarite, aparecen algunos nombres importantes: Hunter (1953), Blau (1955), Coleman, Katz y Menzel (1957, 1966), Coleman (1961), Laumann (1966). Se hacen también progresos decisivos sobre todo en el campo de los métodos y de la conceptualización matemática (Cartwright y Harary, 1956; Davis, 1963, 1967). Pero, de todas formas, la teoría de redes sociales no acaba de entrar dentro de la sociología ortodoxa (J. Galaskiewicz y S. Wasserman, 1993).

Al final de los sesenta se da una ruptura importante con las corrientes mencionadas anteriormente a partir del llamado estructuralismo de Harvard, protagonizado por la escuela de White (1963), Boyd (1969), Lorrain y White, (1971). White establece el análisis de las redes como un método de análisis estructural llegando a esta concepción a partir de modelos algebraicos, la teoría de grafos y el desarrollo de técnicas como la escala multidimensional. 
Granovetter y Lee, aunque aigo aparte de las primeras discusiones de la escuela de Harvard, realizan estudios que, sin ser explícitamente algebraicos tienen una importancia decisiva. Granovetter en Getting a Job (1974), analiza la forma de transmisión de la información en la búsqueda de trabajo y, sobre todo, los lazos que se establecen. Lee en The Search for an Abortionist (1969) estudia cómo la mujer adquiere la información necesaria para abortar.

\section{Los años setenta y ochenta}

Como hemos visto, ya desde Moreno en 1934, el concepto de red social inspira diversos temas de estudio y también en los posteriores años cincuenta y sesenta. Pero es sobre todo a partir de los setenta que proliferan las temáticas y los conteridos de investigación que utilizan la perspectiva y los métodos de las redes sociales como orientación e instrumento de análisis (R.D. Alba, 1982). Antes de los setenta se realizaron numerosas tentativas para diseñar métodos, estudiar minuciosamente las relaciones sociales y descubrir sus pautas aunque muchos de estos intentos fueran relativamente rudimentarios y no condujeran a métodos suficientemente atractivos y de sencilla comprension para los investigadores. En buena medida todo cambia en los últimos sesenta y en los setenta con un mayor desarrollo de la base matemática, concretamente de la teoría de grafos (Harary y orros, 1965; Harary, 1969). La llegada de los algoritmos de computación hace además posible su implantación práctica.

Los años setenta marcan así un gran momento de crecimiento de la teoría de redes: nacimiento de la INSA en el 78, de la revista Social Networks así como muchas investigaciones y su bibliografía correspondiente. Se puede decir que en estos años adquiere la teoría de la redes su mayoría de edad (D. Knoke y J.H. Kuklinski, 1982; J. Galaskiewicz y S. Wasserman, 1993). Los cientificos sociales se ven también atraídos por un tipo de análisis que presenta aspectos de la estructura social que quedaban un tanto abstractos en otras perspectivas (R.D. Alba, 1982).

Las innovaciones más importantes se llevan a cabo en los campos metodológico, teórico y conceptual, por un lado, en el de los métodos, algoritmos $y$ técnicas, por otro, $y$, por fin, aunque con menor intensidad en el de la recogida de datos y del muestreo.

1. En el campo metodológico, teórico y conceptual: en estos años las redes llegan a una cierta plenitud teórica como cuerpo específico de métodos. La teoría de las redes sociales abre nuevos marcos de referencia o la reinserción en ella de teorías existentes. Se dan también infuencias con otras teorías, p.e. la teoría del intexcambio y la teoría de la elección racional. Además, aunque el análisis de redes es para algunos sólo una orientación teórica particular sobre la manera de entender la estructura de los fenómenos sociales vinculada a las teorías estructurales de la acción, para otros constituye una teoría en sentido estricto. 
2. Métodos, algoritmos y técnicas: el avance en los métodos, algoritmos y técnicas ha sido importante sobre todo en la aplicación del formalismo general matemático. A partir de la necesidad de identificar subgrupos de actores a distancias dadas dentro de la red, de agruparlos y de justificar por qué un conjunto de actores están en el mismo subgrupo, etc. (J. Galaskiewicz y S. Wasserman, 1993), se han desarrollado técnicas de análisis estadístico para datos relacionales y han proliferado procedimientos y programas de ordenador para analizarlos.

3. Recogida de datos y del muestreo: se han llevado a cabo trabajos pioneros sobre la recogida de daros y se han usado métodos de muestreo para describir la estructura global, p.e. para estudiar su densidad, la reciprocidad de los lazos. También se han hecho intentos de obtener muestras de lazos relacionales. Aunque hay que decir que los resultados en este sentido no han sido muy eficaces.

Pero, los años setenta aunque han supuesto el inicio de la puesta de largo de la teoría de redes también ha dado muestras de signos de inmadurez, manifestada sobre todo por la escasez, al final de la década, de estudios sustantivos importantes basados en datos y métodos de redes y en la acusada predominancia de cuestiones y contenidos metodológicos en su literatura (R.D. Alba, 1982). Los mayores progresos se han dado en los métodos, ya que al principio de la década se limitaban a un abanico de procedimientos un tanto eclécticos e inciertos en sus resultados. Esta situación acaba en buena medida a finales de los setenta. Los procedimientos analíticos ganan en sofisticación y potencia de análisis, aunque la profusión de los mismos ha podido estar al origen de una cierta confusión para los no iniciados en la literatura de las redes. Los diversos métodos y modelos existentes, sin duda potentes, son aún deudores del marco específico teórico y de aplicación en el que fueron creados. Además hay otra fuente de confusión que proviene de la existencia concurrente de dos importantes tradiciones: la tradición más sociométrica, que viene de Moreno, con base psicologizante y centrada más bien en pequeños y bien limitados grupos y otra tradición más reciente que ve las redes como un medio para estudiar fenómenos más estructurales y macroscópicos.

Es de gran interés seguir la exposición histórica que hacen $\mathrm{S}$. Wasserman y K. Faust (1994:10-16) de las redes sociales a partir de las tres motivaciones que confluyen, según ellos, en su creación y desarrollo: las motivaciones empíricas, las teóricas y las matemáticas.

\section{Aplicaciones}

Veamos algunos campos o temáticas de análisis en los que las redes han mostrado su eficaz aplicación (D. Knoke y J.H. Kuklinski, 1982).

Los estudios sobre organizaciones a pequeña escala han sido muy abundantes: clases escolares y escuelas, despachos, bandas o grupos, clubes sociales, laboratorios u otros lugares de trabajo. La ventaja es que en este tipo de estudio se dan límites claros al poder enumerar los individuos. También se han 
realizado estudios de organizaciones de más envergadura: élites de comunidades, redes en la economía nacional, redes de científicos en una disciplina profesional y relaciones internacionales. Dentro del marco organizacional y/o institucional las redes han tratado las relaciones de poder y autoridad sobre todo en organizaciones complejas formales, p.e.: las redes entre empresas a partir de la presencia en consejos de administración.

Las relaciones de afinidad y de amistad han sido también una materia habitual de las redes sociales; idénticamente las de familia y parentesco, p.e. de roles entre miembros de las familias, matrimonios.

Otra temática corriente se refiere a las relaciones de comunicación: lazos entre actores como canales de transmisión de mensajes; de transacción: actores que intercambian instrumentos físicos o simbólicos, regalos, venta económica, compras, servicios, información sobre trabajo, aborto, consejos políticos, trabajo social.

\section{Definicióni qué son las redes sociales}

\section{Definición y representación.}

Las Redes Sociales pueden definirse como un conjunto bien delimitado de actores -individuos, grupos, organizaciones, comunidades, sociedades globales, etc. - vinculados unos a otros a través de una relación o un conjunto de relaciones sociales. Mitchell (1969:2) añade que las "caracrerísticas de estos lazos en tanto que totalidad pueden ser usados para interpretar los comportamientos sociales de las personas implicadas», pero parece más bien un objetivo genérico que un criterio específico de definición. Otras definiciones son más instrumentales o más centradas en el aparato metodológico, como la de Freeman (1992:12): «colección más o menos precisa de conceptos y procedimientos analíticos y metodológicos que facilita la recogida de daros y el estudio sistemático de pautas de relaciones sociales entre la genten. El rasgo más característico de las redes sociales consiste en que requieren "conceptos, definiciones y procesos en los que las unidades sociales aparecen vinculadas unas a otras a través de diversas relaciones» ( $\mathrm{S}$. Wasserman y K. Faust, 1994:6).

Se pueden dar diversas maneras de formalizar y medir los datos y el análisis de las redes sociales, las dos más importantes: la teoría de los grafos, operando a partir de productos cartesianos con los grafos como representación, y la teoría matricial, a partir de las sociomatrices como matriz de datos inicial.

\section{Conceptos fundamentales en el análisis de las redes sociales}

S. Wasserman y K. Faust (1994: 17-20), los circunscriben a los siguientes:

1. Los actores sociales: son entidades sociales sujetos de los vínculos de las redes sociales. Son de diverso tipo: individuos, empresas, unidades colectivas sociales, departamentos en una empresa, agencias de servicio público en la ciudad, estados, etc. 
2. Los lazos relacionales: son los vínculos entre pares de actores, unidad de análisis en las redes sociales. Son de muy diverso tipo: personales - amistad, respeto, consejo, etc.--; transferencias de recursos — bienes, dinero, información, etc.-; asociaciones, interacciones comportamentales; movilidad geográfica o social; conexiones físicas; relaciones formales u organizacionales; etc.

3. Díada: es la relación específica entre dos actores. Es inherente al par y no se piensa como propiedad de un solo actor. Una díada consiste en un par de actores y el posible lazo entre ambos.

4. Tríada: es el conjunto de tres actores y sus relaciones. Permite el análisis de balance y también el considerar propiedades transitivas.

5. Subgrupo: es una extensión de los conceptos anteriores. Subgrupo de actores es cualquier subconjunto de actores además de los lazos existentes entre ellos.

6. Grupos: las redes sociales tienen además capacidad de modelizar relaciones entre sistemas de actores que denominamos grupos en tanto que conjunto de todos los actores sobre los que se miden los lazos. Se trata siempre de un conjunto finito.

\section{Forma y contenido}

Cabe hacer en las redes una distinción entre forma y contenido. La aproximación de las redes, como muchos otros mćtodos en sociología, es un procedimiento de análisis formal, evidentemente sobre una base sustantiva de relaciones. Se recoge la información, se transforma en dato, se trata formalmente y se interpreta sustantivamente. Ambas caras, forma y contenido, son indisociables en el análisis de redes.

1. El contenido es la materia, la sustancia relacional —afecto, información, dinero, etc. - que "fluye" a través de las unidades por medio de las relaciones que se dan entre ellas a partir del intercambio de dicho contenido. Constituye la materialidad sociológica de la relación. Evidentemente implica, y es lo que realmente interesa, un tipo de comportamiento o acción, e incluso percepción de tal relación, entre dos unidades. Estas relaciones tienen interés para el sociólogo ya que han sido elegidas como problemática de investigación: una unidad tiene a la otra como objeto o efecro directo o indirecto y/o recíproco. No entraremos en la discusión (L.C. Freeman, 1992) de si han de considerarse como sociales todas las relaciones o solamente algunas, p.e., únicamente las conscientes, como sugiere Weber (1947:88), o solamente las consensuadas normativamente, en la visión de Parsons y Shils (1951:195). Con todo, hay un consenso sobre la unidad mínima, la persona, y sobre el vínculo o relación entre ellas como unidad básica de la red y del análisis (Wallace, 1969:5).

Las relaciones pueden ser formales en el sentido de institucionales/ regladas o informales; permanentes o pasajeras; en proceso o consumadas; 
direccionales o no; superficiales o profundas; conscientes o inconscientes. Todo dependerá de la naturaleza del problema analizado (R.D. Alba, 1982). Aunque las relaciones pueden ser muy diversas y múltiples - para una clasificación de esquemas de estas relaciones ver Michell (1973) y Tichy y Fombrun (1979) - , sin embargo el análisis se puede focalizar solamente sobre una o algunas de estas relaciones.

2. Por forma de la red se entienden la expresión abstracta de la relación y las propiedades de la configuración global o de algunas de las partes, es decir, lo que se suele describir como pautas, modelo o estructura de la red. Un ejemplo de tal propiedad formal es la densidad que se define como la proporción de las relaciones de hecho sobre las relaciones potenciales.

Es importante insistir en que una precisa definición conceptual de la relación y su correspondiente expresión formal están a la base de la posibilidad que las redes tienen de modelizar las relaciones y validar los modelos correspondientes. S. Wasserman y K. Faust (1994:11) citan a Leinhardt: "no es posible construir teorias explicativas efectivas usando metáforas».

Aunque el poder de las redes se vincula al potencial explicativo de las propiedades formales ninguna de estas dos dimensiones, sustantiva y formal, ha de ser vista y sobre todo interpretada independientemente, si bien el proceso de análisis se focaliza en los aspectos formales.

\section{Idea central de las redes sociales}

La idea central del análisis de redes reside en el supuesto de que lo que la gente siente, piensa y hace tiene su origen y se manifiesta en las pautas de las relaciones situacionales que se dan entre actores oponiéndose así a la idea de que los atributos o las características de los actores individuales están a la base o son causa de las pautas de comportamientos y, por tanto, de la estructura social. La raza, la edad, el sexo, la categoría social importan mucho menos en la teoría de redes que las formas de las relaciones, mantenibles o mantenidas. Son las relaciones, los vínculos que mantienen los actores, los que establecen las estructuras en cuyas posiciones se sitúan las unidades. Por consiguiente, la explicación de los comportamientos requiere un análisis de cómo los actores están conectados unos a otros en las diversas situaciones en las que son observados. El análisis de redes no es más que un conjunto conceptual y de métodos descriptivos, estructurales y predictivos para conseguirlo (STRUCTURE, versión 4.2., 1991).

Veamos algunas ideas o principios centrales en la teoría de redes sociales independientemente de las diferencias que puedan darse entre los distintos analistas (S. Wasserman y K. Faust, 1994:4).

1. Los acrores y sus acciones son contemplados como interdependientes y no como independientes o unidades autónomas. 
2. Los lazos relacionales entre los actores vehiculan transferencias de recursos, tanto materiales como no materiales.

3. Los modelos contemplan las estructuras de relaciones como entornos que o bien proporcionan oportunidades o bien coaccionan la acción individual.

4. Los modelos de redes identifican la estructura social, económica, política, etc. como pautas constantes de relaciones entre actores.

\section{La perspectiva relacional de las redes sociales}

\section{y la individuadista-atributiva. Datos, lenguajes y niveles de tratamiento}

Insistamos en algunos aspectos del apartado precedente. Para ello volvamos a las diferencias existentes entre el tratamiento que habitualmente se da a los estudios empíricos de la estructura social dentro de la perspectiva atributiva propia, p.e. de las encuestas -claramente reflejados y ejemplificados en todos los artículos de este número de Papers - y la aproximación de las redes sociales. Ambas no son excluyentes, aunque la mayor parte de las investigaciones en el campo empírico sean atributivas. Y no son excluyentes porque muchos aspectos del comportamiento social pueden tratarse de ambas maneras, relacional o atributivamente. Pero para ello se requiere un sutil, aunque importante, cambio de perspectiva (G. Rizzert, 1992, 1993).

No olvidemos que las redes sociales centran su análisis sobre la relación entre las unidades que actúan: todo, es decir, teoría, modelos, métodos y aplicaciones son expresables en términos relacionales de conceptos o procesos.

La diferencia fundamental entre explicaciones dentro de la teoría de redes sociales y otras perspectivas consiste en la inclusión de conceptos y de información sobre las relaciones entre unidades, siendo dicha relación la unidad de análisis fundamental. Así pues, la unidad de análisis en la aproximación de las redes sociales no es el individuo sino el conjunto formado por los individuos y los lazos entre ellos.

En la visión atributiva y atomista los atributos observados de los actores, variables, se entienden en términos de pautas o estructuras entre unidades. En la visión relacional los acributos son secundarios, las relaciones, primarias.

\section{La visión atomista y atributiva versus la visión relacional de la teoria de redes}

\section{La visión atomista y atributiva}

En esta visión el actor o agente, unidad de análisis, es descrito o diseñado en una perspectiva individualista o bien emitiendo opiniones independientemente de los otros, o bien realizando una acción intencional basada en cálculos racionales de maximación de utilidades, o bien actuando según motivaciones predeterminadas por causas antecedentes constrifientes y/o socia- 
lizadas que explican sus acciones individuales. En general no se consideran los contextos sociales en los que el accor social está implicado e inmerso. En los análisis individualistas raramente la interacción del individuo con la estructura social de pertenencia es vista como foco explícito de investigación $y$, por tanto, de toma de datos.

En esta perspectiva las propiedades de los sujetos, p.e.: sexo, edad, inteligencia, ingresos, ocupación, opciones personales, etc., son vistas como características intrinsecas de las unidades e independientes de las relaciones con otros miembros $y$, desde luego, como acabamos de afirmar, también independientes del contexto específico en el que se observan. Los atributos persisten en y se trasladan a los diversos contextos en los que está implicado el actor. De esta forma dichos atributos aparecen perfectamente inalterables $\mathrm{e}$ intercambiables entre, p.e.: el sexo en la casa y en la fábrica, la edad en la asistencia a un espectáculo o en situación laboral, etc. No sólo se intercambian las unidades muestrales aleatoriamente sino que el individuo también "pasea" sus cualidades, sociales sí pero "esencialistas", por todos los contextos de su realidad social (D. Knoke y J.H. Kuklinski, 1982). La idea fundamental de la perspectiva atributiva consiste por tanto en que todo actor tiene acceso diferencial, p.e. a recursos - riqueza, poder, información-, dependiendo de las cualidades intrínsecas de los sujetos sociales. El resultado es que la población, no las relaciones, se estructura - se agrupa y se identifica- en determinadas posiciones según la posesión de, o la pertenencia a, determinadas características o combinaciones de los atributos elegidos. Además, dichas agrupaciones y sus pautas estructurales correspondientes se "ejecutan" en el análisis, sea correlacional u otros.

Aunque la sociologia se ha propuesto siempre estudiar las organizaciones, los sistemas, la estructura social, etc., después de la Segunda Guerra Mundial la mayor parte de los trabajos empíricos han estado mayoritariamente focalizados hacia la perspectiva individual y atributiva. En ello han tenido un peso decisivo, como sabemos, la imparable introducción de las clásicas encuestas sociales - que se han convertido en el paradigma de la aproximación atomista y atributiva-, con sus correlatos empiristas de la estadística y de los indicadores sociales además de la posibilidad que ofrecieron, y continúan ofreciendo, las técnicas y los métodos de análisis para ir más altá de los grupos reducidos (J. Galaskiewicz y S. Wasserman, 1993).

EI análisis de las redes ha aparecido un tanto inconfortable a los valedores de la perspectiva atomista y atributiva. No hay duda de que el enfoque atributivo ha producido y producirá informaciones y análisis válidos sobre movilidad social, comportamientos políticos y sociales, actitudes y otras muchas temáticas. Sin embargo ha ignorado que la base de la sociología es la interacción, o la relación entre sujetos o actores sociales y entre posiciones, y que, por tanto, esta base o unidad elemental sociológica debe ser respetada en su integridad en la elección de la problemática, en el modelo de análisis, en la toma de información y, por supuesto, en el análisis. Además, debe recuperarse en la interpretación. 


\section{La visión relacional de la teoría de redes}

La idea central de la visión relacional consiste en que el análisis no se construye tanto a través de categorías sociales o atributos, sino a través de los lazos o vínculos entre actores, incluso no estando directamente relacionados y unidos (Wellman, 1983; Granovetter, 1973).

En la perspectiva relacional las acciones son consideradas en la medida que expresan una(s) propiedad(es) emergente(s) de la conexión o ligazón entre unidades de observación (D. Knoke y J.H. Kuklinski, 1982). Además, las relaciones están contextualizadas específicamente y se alteran o desaparecen según dichos contextos de tal manera que se considera al actor a partir de la interacción con otras partes del contexto de la red o, al contrario, no es considerado si no está incluido en un contexto relacional dado. En las redes la existencia y la intensidad de las relaciones pueden ser también medidas; no olvidemos quc estamos dentro de una sociología "cuantitativa". Se miden, p.e., la fuerza de la relación amical, de los servicios dados en el interior de las familias, de los intercambios económicos entre organizaciones, etc. Las medidas y pautas relacionales pueden captar propiedades emergentes o establecidas del conjunto social estudiado no percibibles como simples agregaciones de los atributos de los individuos. Tales propiedades afectan al conjunto del sistema y al comportamiento de las unidades de la red. En este sentido no es suficiente, p.e., con afirmar que la gente de estatus alto tiene más poder que la gente de bajo prestigio. De lo que hay que dar cuenta o explicar es de como los modelos o pautas relacionales crean diferencias de poder, estatus o prestigio entre la gente.

La teoría de redes cambia el visor de la investigación: de la búsqueda de factores concomitantes o causales a la indagación de como dichos factores pueden producir sus efectos a partir de las relaciones. El supuesto básico de la teoría de redes, ya lo hemos dicho, consiste en que la indudable correlación que se da entre comportamientos, opiniones, etc., y las variables atributivas como sexo, raza, edad, educación, ocupación, etc. - -al origen de las estructuras sociales clásicas en los análisis empíricos a partir de los valores de dichos atributos-, no dejan de ser globalmente espúteas, ya que las verdaderas variables explicativas alternativas son las relaciones (STRUCTURE, versión 4.2, 1991).

\section{Los tipos de datos en función de estos enfoques}

Los tipos de datos pertinentes para las redes serán, por consiguiente, los telacionales y no precisamente los datos atributivos como actirudes, opiniones o variables de hecho, etc., aunque también pueden ser utilizados. Los datos relacionales, o variables estructurales, pueden obtenerse también por cuestionarios, documentos, archivos, por la observación o también por otros métodos etnográficos.

Los datos relacionales expresan contactos, transacciones, lazos, conexiones, vínculos, servicios dados o recibidos, comunicaciones entre grupos a partir de agentes. En definitiva, como venimos insistiendo, conectan pares de agentes entre sí. Precisamente expresan los lazos de funcionamiento entre distintos 
tipos de agentes. El dato es la información y la medida de esta relación (J. Scott, 1991).

\section{Los lenguajes formalizados utilizados}

Como ya decíamos al tratar de los orígenes de las redes sociales la teoría de grafos ha sido de uso habirual. Esta teoría proporciona una representación apropiada y un conjunto de conceptos para estudiar las propiedades formales de las redes sociales. El grafo, presente ya en Moreno, es simplemente una serie de líneas que conectan puntos. Los puntos son las unidades y las líneas son relaciones, dirigidas o no dirigidas. Así se puede estudiar el grupo giobal a partir del punto de vista de cada uno sin centrarse en un solo punto focal.

La teoria de grafos, y su representación, ha suministrado no sólo una forma de visualización, y con ello una cierta familiaridad y popularidad a la teoría de redes, sino que, tomada como teoría matemática, ha proporcionado también conceptos y teoremas para muchos de los indicadores utilizados en las redes sociales. Con todo, la matemática requerida de la teoría de grafos y utilizada para el tratamiento básico o elemental de las redes sociales no es excesiva: se trabaja sobre todo con conceptos de dicha teorf́a y algo menos con su desarrollo en teoremas. La teoría de grafos traduce o traslada los datos en conjuntos formales que pueden estar directamente relacionados con los hechos sustantivos de las redes. El grafo ha suministrado los algoritmos a los programas UCINET y GRADAP.

La teoría de grafos no es la única expresión formalizada utilizada para modelar las redes, aunque haya sido punto de arranque de muchos de sus conceptos fundamentales. La teoría matricial y de los espacios vectoriales, así como la teoría estadística han sido importantes en vistas a validar hipótesis sobre tendencias estructurales. Además diversos modelos matemático-algebraicos han estado presentes en el tratamiento de las redes sociales, p.e.: los modelos de log-linear en el análisis de las interacciones entre díadas. Los modelos algebraico-estadisticos permiten validar proposiciones teóricas sobre redes, calcular errores y usar tests de ajuste (S. Wasserman y K. Faust, 1994:15-16). La teoría matricial, concretamente, está a la base del tratamiento de las Redes, aunque la visualización que consigue no sea tan clara como la de los grafos. Desde luego si las unidades aumentan y las relaciones son más complejas el tratamiento matricial es imprescindible. Con la teoría matricial se utilizan, teorías algebraicas y métodos habituales en el tratamiento multidimensional de la sociologia, espacios vectoriales, distancias y similitudes, análisis factoriales, análisis de cluster, etc., caso del programa STRUCTURE, escalas multidimensionales, así como los conceptos y desarrollos habituales de la estadística aplicada a las ciencias sociales, correlaciones, ajustes, etc.

\section{Los niveles de análisis}

El enfoque de las redes sociales trabaja sobre diversos niveles de análisis. El 
interés de esta aproximación es debido también a la posibilidad de tratarlos conjuntamente (P.V. Marsden, 1990).

El nivel egocéntrico o personal de análisis: hay analistas que tratan la estructura de las redes buscando y explicando las diferencias entre actores por sus posiciones sociales provenientes de las redes locales que rodean a los actores y que, en cierto sentido, les "pertenecen". Este diseño no conduce a una descripción completa de la estructura social de una población, a menos que las unidades estén ya redefinidas como posiciones sociales generales tales como grupos ocupacionales o étnicos. Por contra y como ventaja, este enfoque se presta a utilizar mutestras representativas y es compatible con los métodos de la estadística tradicional para la generalización de resultados a amplias poblaciones.

El nivel intermedio de análisis: los estudios de las redes pueden reducirse a un nivel intermedio al pretender analizar las relaciones de las unidades entre sí, de las unidades con parte de la red o con la red total. A menudo lo que se analiza son díadas pero pueden también ser estudiados subconjuntos más amplios. Se ha tratado también de realizar directamente el muestreo aleatorio de díadas o tríadas, aunque no es habitual.

La referencia mayor y de más interés es el nivel de la estructura completa. Lo más pertinente consiste en comparar entre sí estructuras completas sociales p.e.: de trabajo, grupos, organizaciones, comunidades. Requiere datos completos de redes sobre los lazos sociales que vinculan los elementos de la población o de una población con otra. La enumeración completa de la población relacionable es esencial para las técnicas analiticas que usen información sobre lazos indirectos o los compuestos que ligan los actores. Tal diseño es necesario para estimar algunas propiedades estructurales o para técnicas tales como los análisis de modelos de bloques a fin de identificar los roles y las posiciones basados en los conceptos de equivalencia estructural, que comentaremos postcriormente. Hay otros métodos para este nivel de estudio, p.e., de centralidad (Freeman, 1979) y algunos tipos de análisis posicional (Winship y Mandel, 1984).

$\mathrm{El}$ análisis de redes desarrolla un nivel descriptivo de explicación de los fenómenos sociales proporcionando un vocabulario y un conjunto de definiciones formales para expresar conceptos teóricos y propiedades. Pero también puede ser usado para validar y evaluar teorías sobre los procesos relacionales y estructurales. Tales teorías proponen resultados específicos estructurales que pueden ser contrastados con los datos observados ( $\mathrm{S}$ Wasserman y K. Faust, 1994:5).

\section{Las redes, un enfoque estructural}

El enfoque de las redes es estructural. No se entra aquí en la naturaleza de dichas estructuras, de si son larentes o manifiestas; objetivas, autónomas, coercitivas y exógenas a los sujetos sociales o, al contrario, inherentes a ellos; construidas simbólicamente, antes, durante o después de la interacción o inte- 
riorizadas vía socialización. Tampoco se entra en la variedad y el contenido de los distintos estructuralismos como corrientes de pensamiento en sociología. Las redes sociales acostumbran a adoptar una perspectiva pragmática y empirica en la consideración y el tratamiento del concepto de estructura. A los pensadores de las redes sociales les ha interesado menos filosofar y más la realización de análisis metodológicos rigurosos, empíricos o formales, de los diversos tipos de redes. De todas formas, su punto de vista tiene más que ver con la idea de estructura de la sociología tradicional que con la de Levi-Strauss.

El enfoque de redes concibe la estructura social como pautas, modelos de relaciones específicas que ligan unidades sociales, incluyendo actores individuales y colectivos. Más allá del uso del concepto de red social como simple metáfora y como un puro instrumento de investigación, esta aproximación trata de interpretar el comportamiento de los actores a la luz de sus posiciones variables dentro de la estructura social. Por dicha posición se ponen de manifiesto las constricciones de la estructura social sobre la acción individual y también las oportunidades diferenciales -conocidas como recursos sociales, como capital o como soportes sociales- a las que tienen acceso los actores.

Dado un conjunto de actores, el análisis de redes sociales trata las variables estructurales medidas sobre los actores considerados como tal conjunto. Precisamente define la estructura relacional de un grupo o de un sistema más amplio como las pautas, o la pauta, de relaciones del conjunto de actores del grupo. Los analistas de redes sociales estudian los modelos de estas relaciones para dar cuenta de la estructura del grupo. De esta forma se puede estudiar el impacto o la influencia de la estructura sobre el funcionamiento del grupo y/o sobre los individuos (S. Wasserman y K. Faust, 1994:9).

Wellman y Berkowitz (1991) señalan diversos tipos de errores o malentendidos en el tratamiento de las redes sociales como, p.e., el considerarlas como puro método de análisis vacío de cualquier punto de vista sustantivo. Las redes sociales requieren nuevos hábitos intelectuales, aunque se ha de pensar que la manera estructuralista de contemplar e interpretar los fenómenos sociales también se adecua y está presente en nuestra experiencia ordinaria: siempre nos situamos y situamos a los otros en conjuntos de similitudes y diferencias cuando conocemos $e$ interpretamos las relaciones que mantienen: quien trabaja en el mismo sitio que otro, etc. Los analistas estructurales hacen lo mismo pero más abstractamente y a escala más amplia. Pensar estructuralmente requiere que pongamos atención a las pautas de relaciones que se dan en conjuntos humanos, instituciones u organizaciones.

Para Wellman el análisis estructural, análisis de redes, se alimenta en una serie de consideraciones básicas, que llama paradigmas, que proporcionan la unidad intelectual del análisis: 1) la idea de que los comportamientos de las unidades (individuos, grupos, etc.) han de ser interpretados más bien en términos de constricciones estructurales sobre la actividad de dichas unidades que en términos de fuerzas internas existentes dentro de las unidades adquiridas, p.e., vía socialización de normas; 2) que los análisis no se han de hacer tanto a través de la clasificación de unidades por sus atributos sino más bien a partir de 
las relaciones que mantienen; 3) que son precisamente las relaciones pautadas las que afectan a los comportamientos de los miembros de las redes traduciéndose en que las poblaciones y las muestras se han de definir relacionalmente; 4) que los métodos categoriales de descripción y análisis atributivos han de ser reemplazados por métodos relacionales; 5) que la estructura puede también ser considerada como una red de redes pudiendo a su vez ser, o no, subdividida más discretamente sin asumir así la impenetrabilidad de los grupos; 6) que los métodos analíticos han de usar directamente la naturaleza relacional y las relaciones pautadas de las estructuras sociales como unidades de análisis, lo que quiere decir que se ha de utilizar menos una perspectiva individualista y más una matemática y estadística que permitan estudiar la estructura social más directamente.

Para Wellman el estructuralismo de las redes sociales es diferente de otros estructuralismos, ya que se focaliza sobre las relaciones sociales concretas entre actores sociales específicos y porque pone de relieve el intercambio que to aproxima más, según él, a los input-output económicos y a la física cuántica que al estructuralismo de Levi-Strauss.

Wellman establece cinco principios generales que han de guiar el trabajo analítico estructural de las redes sociales en una gran variedad de áreas sustantivas:

1. Las relaciones sociales estructuradas son una fuente más poderosa de explicación sociológica que los atributos personales de los miembros de un sistema. Wellman hace un desarrollo exhaustivo sobre las diferencias entre el análisis atributivo y el relacional que ya se ha comentado.

2. Las normas emergen de la localización de las relaciones sociales en los sistemas estructurados.

3. Las estructuras sociales determinan la operación de las relaciones diádicas o relaciones a pares.

4. El mundo está compuesto de redes, no de grupos.

5. Los mérodos estructurales complementan y suplen los métodos individualistas.

El desarrollo que Burt hace de las redes como aproximación estructural difiere evidentemente de toda visión normativa y atomista. Burt parte de la existencia del conjunto estacus/roles de los actores generados por la división del trabajo. Un actor evalúa la utilidad de las acciones alternativas, en parte, en función de las condiciones personales y, en parte, en función de las condiciones de los otros. De esta manera los actores son, para Burt, intencionales bajo las constricciones de la estructura social (R.S. Burt, 1982:8, 9).

El esquema de Burt, muy repetido, es el siguiente,

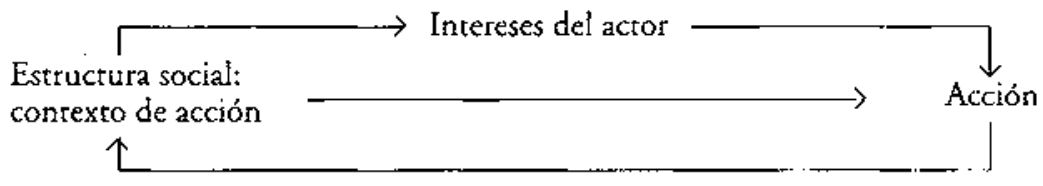


La teoría de redes incorpora para Burt dos supuestos básicos y significativos. El primer supuesto es que todo actor social participa en un sistema social que implica otros actores que son puntos de referencia significante en las decisiones mutuas. Las relaciones que un actor mantiene con otros pueden afectar a sus acciones, percepciones y comportamientos. La distribución desigual condiciona tanto la cooperación, lo que implica colaboración entre individuos, como la competencia, lo que implica conflicto. Esto da una naturaleza dinámica a la teoría de redes, ya que la estructura del sistema se modifica conforme a las pautas cambiantes de la coalición y el conflicto. El segundo supuesto es que no se puede detener el análisis en el comportamiento social de los individuos. Se ha de llegar a diversos niveles de la estructura del sistema social. La estructura son regularidades de relación entre entidades concretas (D. Knoke y J.H. Kuklinski, 1982).

El tratamiento o perspectiva de las redes añade, en la búsqueda de la estructura relacional, valores a la investigación como eficacia, claridad y validez. La eficacia proviene sobre todo de su capacidad para iluminar estructuras sociales completas y para comprender los elementos particulares de las mismas. Además, en la teoría de redes la "fuerza causal" se origina en la estructura relacional que define una situación. La claridad proviene de tener que explicitar qué tipo de relación se combina para generar la "fuerza causal». No es suficiente con decir que determinada gente similar socialmente, p.e. los de una categoría o grupo socioeconómico, tiene unas características de comportamiento porque pertenecen a tai categoría o grupo, sino que lo que hay que decir es como las relaciones, única realidad social de base, producen una estructuración tal que da la similirud de comportamientos. La validez del efecto directo relacional y la superacion de todo posible efecto espúreo de las variables atributivas proviene de tener que explicitar qué relaciones se agregan para crear la fuerza causal ya que, precisamente, se han rechazado los atributos como variables causales (STRUCTURE, versión 4.2., 1991).

\section{Perspectivas o modelos de tratamiento en las redes sociales,} criterios (intuiciones) para analizar la estructura relacional y principios de análisis

\section{Perspectivas o métodos y criterios de cohesión social $y$ de estructura de equivalencias}

Existen diversos métodos de análisis, de ahí la riqueza que supone la urilización de las redes para el tratamiento de los datos relacionales. Lo que más puede interesamos dentro de la visión general que se persigue en este articulo es entrar en las dos perspectivas de tratamiento ya anunciadas que han creado escuela dentro de las redes sociales: la relacional y la posicional (Burt, 1978, 1980b.; R.D. Alba, 1982).

Veamos también, después de dar cuenta de dichas aproximaciones, cuales son los criterios que a manera de intuiciones guían el análisis en la aplicación 
de dichas perspectivas a la hora de identificar las posiciones y determinar los actores que ocupan cada una.

\section{Caractetización de las aproximaciones}

a) Los métodos relacionales se basan en conexiones directas e indirectas que existen entre unidades de una red y están más focalizados hacia el análisis micro poniendo más el énfasis sobre subgrafos de pares ordenados, díadas y tríadas. La cohesión es la palabra clave.

b) Los posicionales se basan sobre las similitudes en las pautas de las relaciones de unas unidades con otras. Se centran más en el análisis macro, en particular en la descripción de estructuras sociales globales. En la perspectiva posicional la semejanza entre conductas se debe a la equivalencia estructural. Es decir, al hecho de que los actores estructuralmente equivalentes tienen relaciones idénticas con el conjunto de los otros actores. Puede haber equivalencias estructurales entre corporaciones aunque no haya relación directa o indirecta entre ellas, cosa que es impensable en la perspectiva relacional. Las unidades que están en la misma posición en la estructura se comportan del mismo modo porque mantienen una relación idéntica con otra u otras dentro de la red total. La equivalencia estructural juega aquí el papel clave en la explicación de la semejanza de las conductas.

El enfoque posicional está más vinculado a Burt y White, que proporcionaron el algoritmo básico del modelo. Se percataron de que examinando los perfiles de los actores en la red se podían descubrir conjuntos de roles diferentes dentro de un sistema social (J. Galaskiewicz y S. Wasserman, 1993).

2. Criterios que a manera de intuiciones guían el análisis en la aplicación de dichas perspectivas

La diferencia de los enfoques, relacional y posicional, tiene en cuenta una difcrencia fundamental a la hora de concebir la vinculación entre actores. Mizruchi (1990: 21, 25), habla de dos conceptos de cohesión: subjetiva y objetiva.

a) La cohesión subjeriva, en la línea más relacional, tiene una función de identificación o asociación de los miembros del grupo con los de su grupo, cn particular a partir del sentimiento de que los intereses individuales están ligados a los intereses del grupo. Ello implica la acentuación del sistema normativo. La cohesión se da, o bien vía internalización del sistema normativo, o debido a la presión ejercida por el grupo.

b) La vinculación de la cohesión objetiva, quizás en la línea más posicional, es considerada como proceso objetivo observable independiente de los sentimientos de los individuos (G. Ritzert, 1992, 1993). 
Burt (1978), presenta también estas dos alternativas.

a) El primer criterio, más bien en la perspectiva relacional, es el de cohesión social. Por él los actores están agregados y juntos en una posición en la medida en que están conectados más directamente a los otros por lazos o vínculos (cohesivos).

b) El segundo criterio, más bien en la perspectiva estructural o posicional, es el de la estructura de equivalencia (Lorrain y White, 1971; White y otros, 1976). Este criterio ha sido definido en el sentido de que los actores ocupan posiciones o roles equivalentes en un conjunto debido a los lazos comunes a otros en el sistema. No se requiere que sean lazos directos. (Ver la ilustración extraída de D. Knoke y J.H. Kuklinski, 1982.)'

Las diferentes maneras de identificar posiciones estructurales llevan a diferentes resultados. La elección dependerá básicamente de la elección metodológica y del problema sustantivo y teórico investigado. Para algunos propósitos la aproximación relacional será preferible, mientras que en otras situaciones la de posiciones equivalentes será más interesante.

\section{Fenómenos y principios que abarcan dichos criterios}

Los fenómenos inherentes a las redes sociales, y por tanto objeto también de análisis, son fundamentalmente tres: los límites de las redes, los lazos y los comportamientos (STRUCTURE, versión 4.2., 1991).

Los principios que corresponden a los modelos y a los criterios para el análisis de estos fenómenos son, además de los de cohesión y equivalencia estructural ligados a los criterios precedentes, otros como de prominencia, rango y corretaje.

Estos principios definen los diferentes tipos de interpretaciones que se pueden dar de los fenómenos sociales a partir de las redes sociales. Cada princi-

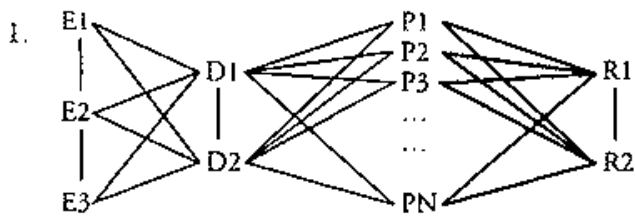

Por el primer criterio, de cohesión, se identifican dos ucliques": una pequeña de los dos recepcionistas y otra más amplia de los doctores y enfermeras. Por el segundo de los criterios, el de equivalencia, hay cuatro posiciones diferentes correspondientes a los cuatro roles del diagrama: enfermeras, doctores, pacientes y recepcionistas. Precisamente los docrores y las enfermeras no están agregados, pues difieren en roles de los otros, los doctores están vinculados a los pacientes y las nurses no lo están. Tres de las posiciones de equivalencia estrucrural son a su vez cliques, posiciones de cohesión, pero la posición del paciente no lo es de clique pues los pacientes no discuten de cuestiones medicinales entre sí (D. Knoke y J.H. Kuklinski, 1982). 
pio implica una intuición estructural, un moror implícito dentro del comportamiento y creencias y un modelo teórico que agregan relaciones observadas en imágenes estructurales ${ }^{2}$. (Ver en nota 2 un gráfico aclaratorio para todos ellos.)

- Principio de cohesión. Como hemos dicho precedentemente, la intuición estructural que alimenta este principio consiste en que los actores similares lo son en la medida en que están vinculados por relaciones entre ellos. Dichas relaciones son además responsables de los procesos de socialización por interacción y por ello de la similaridad de creencias y tendencias comportamentales. La fuerza causal de los comportamientos se basa precisamente en la intensidad de los lazos de comunicación. Es un principio que nos marca también las fronteras del grupo.

- Principio de equivalencia. Como también hemos comentado, la intuición estructural que está en la base de este principio es que actores equivalentes son los que poseen pautas de relación semejantes a los de su misma posición con relación a otros actores. El modelo define los límites sociales de posiciones distintas y origina creencias y tendencias comportamentales homogéneas entre actores equivalentes. La fuerza causal está vinculada al rol jugado por la posición dentro del armazón de referencia compartido (Lorrain y White, 1971; White, Breiger y Boorman, 1976; Burt, 1976 , 1977, 1982, 1987; Winship y Mandel, 1984).

Los principios que vienen a continuación, de prominencia, de rango y de corretaje, predicen el comportamiento midiendo el poder o la libertad de un actor a actuar. Los dos últimos, de rango y corretaje, miden más directamente su potencial de acción difiriendo del primero, de prominencia, en el senti-

2. Principios de:

Cohesión

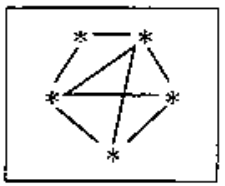

Prominencia,

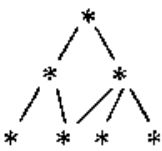

Equivalencia

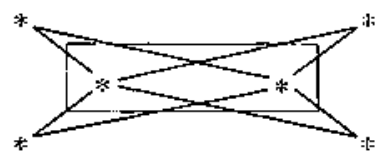

Rango,

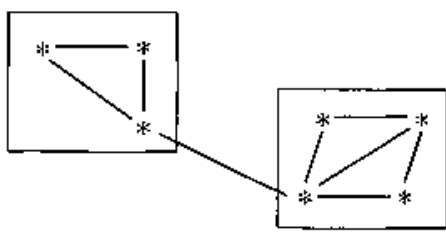

Intermediatio

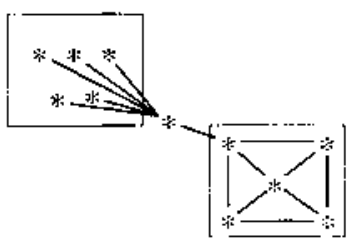


do de que los individuos tienen posibilidad de evitar o negociar los esfuerzos de control de los otros.

- Principio de prominencia. El principio de prominencia sitúa a los individuos sobre un eje vertical a mantera del organigrama de una organización: los líderes arriba, los dirigidos abajo. Se invoca el mecanismo causal en la medida en que un individuo es el objeto de las relaciones de muchos otros, que son a su vez objeto de las relaciones de otros, etc. Este principio supone la "utilización» de otros por parte del líder de la red a fin de impulsarlos a que lleven a cabo sus deseos u órdenes (Hubbel, 1965; Coleman, 1966, 1972; Marsden, 1984).

- Principio de rango. El principio de rango se aplica de una manera simple como es, p.e., la suma de las relaciones que posee un actor: más relaciones se poseen, más acceso se tiene a los recursos sociales. Pero se pueden encontrar modelos más sofisticados como los vínculos de puente. Las relaciones de puente son un importante recurso social. La fuerza causal se encitentra precisamente en esta posibilidad de acceso relacional para evaluar y utilizar recursos (Granovetter, 1973).

- Principio de intermediario (Brocker). Bajo este principio un actor individual riene la libertad de seguir o perseguir sus propios intereses en la medida en que sus relaciones conecten con gente que está desorganizada, pudiendo contraponer unos contra orros, librándose él, de esta manera, de dichas constricciones. La fuerza causal reposa en el contacto que se mantiene con los otros que están desorganizados (Freeman, 1977; Burt, 1980a., 1980b., 1982).

\section{Reflexiones finales}

Hemos prerendido en estas primeras páginas hacer una introducción descriptiva de las cuestiones fundamentales, los objetivos y las temáticas sociales propias a, y que están en el centro de, la teoría de las redes sociales. Volveremos en el futuro con otras contribuciones dedicadas a aspectos más específicos y más vinculados a los métodos y a la práctica de investigación.

Cuando se trabaja en el campo de las redes sociales se tiene la percepción no errónea de estar en un ámbito algo lateral y al margen del quehacer general de los sociólogos. Esta sensación es tanto más chocante cuanto que la intuición central, que está a la base de las redes sociales, es la unidad conceptual básica de la misma sociologia: la relación e interacción social. Son diversas las causas que han podido originar tal situación sobre todo en la sociología, entre otras, la diversidad y dispersión de los métodos y modelos empleados, su elevado tecnicismo, su sofisticación y formalización conceptual, la relativa adolescencia en que se encuentra el desarrollo de las redes - aunque su origen se remonte a los cuarenta es en la década de los setenta y ochenta donde se han dado los avances más notorios-. La teoría de redes sociales necesita realizar progresos en aspectos que son decisivos para su "integración" o para que sea considerada como "sociologia». 
Entre los investigadores de las redes sociales se da un consenso sobre las temáticas o los aspectos en los que las redes sociales deberían progresar. He aquí alguno de ellos sin ánimo de exhaustividad ni tampoco de entrar en detalles y tecnicismos. Ha de llegar a ser una teoría, en el sentido de que se consolide como una perspectiva o aproximación más unificada, no sólo en lo referente a los principios e intuiciones sociológicos que están en su base, en buena medida conseguido, sino también en cuanto a los modelos, los métodos y las técnicas que abarca. De alguna manera se ha de llegar a que el atractivo que evoca su nombre, redes sociales, se vea correspondido con una teoría coherente y, si es posible, deductiva. En este sentido es importante que se desarrollen modelos algebraicos y estadísticos más ambiciosos y adaptados a las redes a fin de integrar en ellos los numerosos conceptos que aparecen, a veces, yuxtapuestos. Estos modelos, a su vez, han de desarrollar e incorporar métodos y técnicas de análisis distribucionales con pruebas de ajuste que superen una visión metodológica de las redes sociales excesivamente descriptiva por el momento.

Es importante también un mayor progreso en el estudio de las redes múltiples, o de varias relaciones, como también las redes valuadas, o no dicotómicas, y las direccionales. Es necesario un desarrollo imprescindible en el campo del muestreo, básicamente por la dificultad que existe de compaginar redes completas con redes extensas y representativas, hoy por hoy reducidas solamente a los análisis egocentrados. De favorecerse esta conjugación el salto de popularidad y de propagación que conseguirian las redes sociales sería considerable, tanto para su integración en la sociología corriente como para el manejo y el conocimiento de sus técnicas y su jerga correspondientes. Las redes sociales, que han supuesto una esperanza e innovación en el tratamiento de la realidad social, han de confirmar tal supuesto en los proximos lustros.

\section{Bibliografia}

ALBA, R. D. (1982). "Taking stock of newwork analysis". En Research in the Sociology of Organizations 1:39-74.

BARNES, J.A. (1954). "Class and Commicee in a Norwegian Island Parish". En Humans Relations 7.

- (1969). "Graph Theory and Social Networks. En Sociology 3.

BarneS J.A.; Harary, F. (1983). "Graph Theory in Neetwork Aanlysis». En Social Networks 5.

Bavelas, A. (1948). "A Matematical Model for Group Structure". En Applied Anthropology 7.

- (1950). "Communication Patterns in Task-Oriented Groups". En Jounal of the Acoustical Society of America 22.

BLAU, P.M. (1955). "Dynamics of Bureaucracy: A Study of Interpersonal Relations in Two Goverment Agencies". Chicago: University of Chicago Press.

Boissevain, J.; Mitchell, J.C. (ed.) (1973). Networks Analysis: Studies in Human Interactions. The Hague: Mouton.

Boorman, S.A.; WHITE, H. (1976). "Social Structure from Multiple Networks II. Role Structurew. En American Joumal of Sociology 2:113-120. 
Bort, E. (1955), "Urban Families: Conjugal Roles and Social Nerworks". En Humans Relations 8.

BoTr, E. (1956). "Urban Families: The Norms of Conjugal Roles". En Humans Relations 9.

- (1957). Family and Social Networks. Londres: Tavistock.

BoyD, J.P. (1969), "The Algebra of Group Kinship". En Jounal of Mathematical Socio$\log 6$.

BURT, R.S. (1976). «Posirion in networks». En Social Forces 55: 93-122.

- (1977). "Positions in multiple network systems, Parr One: A general conceprion of stratification and prestige in a system of actors cast as a social topology". En Social Forces 56: 106-131.

- (1978). "Cohesion versus structural equivalence as a basis for nezwork subgroups". En Sociological Methods \& Research 7: 189-212.

- (1980a) «Cooptive corporate actor networks: a reconsideration of interlocking direcrorates involving American manufacturing". En Administrative Science Quarteley 25: $557-582$.

- (1980b) "Models of network structure". En Annual Review of Sociology 6: 79-141.

- (1982). Toward a Structural Theory of Attion: Network Models of Social Structure, Perception and Action. Nueva York: Academic Press.

- (1983). Corporate Profits and Cooptation: Networks of Market Constraints and Directorate Ties in the American Economy. Nueva York: Academic Press.

- (1987). "Social Contagion and Innovation, Cohesion Versus Strucrural Equivalece". En American Journal of Sociology 92: 1287- 1335.

CARTWRIGHT, D. (ed.) (1959). Studies in Social Power. Ann Arbor: Institute for Social Research.

CARTWRIGHT, D.; ZANDER, A, (Ed) (1953). Group Dynamics. Londres: Tavistock.

CARTWRIGHT; D.; HARARY, F. (1956). "Structural Balance: a Generalisation of Heider's Theory". En Psychological Review 63.

Coleman, J.S. (1961). The Adolescent Society: The Social Life of the Teenager and its Impact on Education. Nueva York: Pree Press.

- (1966). "Foundacions for a theory of collective action". En Journal of Sociology 71 : 615-627.

- (1972). "Systems of social exchange". En Journal of Mathematical Sociology 2: $145-163$.

- (1988). "Social Capital in the Creation of Human Capital". En Amercian Journal of Sociology 94: S, 95- 120.

COLEMAN, J.S.; KATZ, E; MENZEL, H. (1957). "The diffusion of an Innovation Among Physicians". En Sociolmetry 20: 253-270.

- (1966). Medical Innovation: A Diffusion Study. Indianapolis: Bobbs-Merrill.

DAvIS, J.A. (1963), "Structural Balance, Mechanical Solidarity, and Interpersonal Relations". En American Journal of Sociology 68: 444- 462.

- (1967). "Clustering and Structural Balance in Graph". En Humans Relations 20: 181-187.

Davis, A.; Gardner, B.B.; Gardner, M.R. (1941). Deep South. Chicago: University of Chicago Press.

FESTINGER, L. (1949). "The Analysis of Sociograms Using Matrix Algebra». En Humans Relations 2. 
FREEMAN, L.C. (1977). "A Set of Measures of Centrality Based on Betweeness». En Sociometry 40:35-41.

- (1979), "Centrality in Social Networks: I. Conceptual Clariftcation". En Social Netwroks 1 .

- (1992). "Social Networks and the Strucure Experiment». En Freeman, L.C.; White, D.R.; Romney, A.K. Research Methods in Social Network Analysis. New Brunswick (USA), Londres: Transaction Publishers, p. 11-40.

FreEMAN, L.C.; WHITE, D.R.; ROMNEX, A.K. (ed.) (1992). Research Methods in Social Network Analysis. New Brunswick. (USA), Londres: Transaction Publishers.

GalaSKIEWTCZ, J. (1979). Exchange Networks and Community Politics. Beverly Hills: Sage.

GalaskifwicZ, J.; WASSERMAN, S. (1993). "Social Network Analysis. Concepts, Methodology, and Directions for the 1990s". En Sociological Methods \& Research. Vol. 22, 1:3-22.

Granoverter, M. (1973). "The Strengh of Weak Ties". En American Jounal of Socio$\log y 78$.

- (1974). Getting a job. Cambridge, Mass.: Harvard University Press.

- (1982). "The Strenght of Weak Ties: A Network Theory Revisited". En Marsden, P.V.; Lind, N. Social Structure and Network Analysis. Beverly Hills: Sage.

- (1985). "Economic Action and Social Structure: The Problem of Embeddedness". En American Journal of Sociology $91: 481-510$

HaRARY, F. (1969). Graph theory. Reading Mass.: Addison-Wesley.

HARARY, F; NORMAN, R.Z. (1953). Graph Theory as a Mathematical Model in Social Science. Ann Arbor, Mich: Inscitute for Social Science.

HaRARY, F.R.; Norman, Z.; CarTwRIGHT, D. (1965). Structural Models: $A$ intraduction to the Theory of Directed Graphs. Nueva York: John Wiley.

HEIDER, F. (1946). "Attitudes and Cognitive Orientation". En Journal of Psychology 21.

HubBel, C.C. (1965). "An input-output approach to clique identification". En Sociometry 28: 377-399.

HUNTER, F (1953). Community Power Structure: A Study of Decision-Markers. Chapel Hill: Universiry of Carolina Press.

KNOKE, David; KUKLINSKI, James H. (1982). Network analysis. Newbury Park, Londres: Sage.

Laumani, E.O. (1966). Prestige and Association in an Urban Community: An Examination of an Urban Stratification System. Indianapolis: Bodds-Merrill.

LEe, N.H. (1969). The Search for an Abortionist. Chicago: Unjversity of Chicago Press.

LEINharot, S. (ed.) (1984). Social Networks: A Developing Paradigm. Nueva York: Academic Press.

LEwin, K. (1936). Principles of Topological Psychology. Nueva York: McGraw-Hill.

- (1951). Pield Theory in the Social Science. Nueva York: Larper.

LORRAIN, E; WHITE, H. (1971). "Structural Equivalance of Individuals in Social Networks». En Joumal of Mathematical Sociology 1.

MARSDEN, Peter V. (1984). "Restricted access in networks and models of power". En American Journal of Sociology 88: 686-717.

MARSDEN, Peter V. (1990). "Network data and Measurement". En Annual Reviews Sociological 16:435-463.

MARSDEN, PV.; LiND, N. (ed.) (1982). Social Structure and Network Analysis. Beverly Hills: Sage. 
MITCHELL, J.C. (ed.) (1973). «Networks, norms and institutions». En Botssevain, J.; MitcheII. J.C. (ed.) Networks Analysis: Studies in Human Interactions. The Hague: Mouton.

- (1969). Social Networks in Urban Settings. Manchester, Inglaterra: Manchester University Press.

MizRUCHI, M. (1990). "Cohesion, Structural Equivalence and Similariry of Behavior".

En Sociological Theory 8: 16-32.

Moreno, J. (1934). Who Shall Survive? Nueva York: Beacon Press.

NADEL, S.F. (1957). The Theory of Social Structure. Londres: Cohen and West.

NEwCOMB, T.M. (1961). The Acquitance Process. Nueva York: Holt, Renihart y Winston.

Parsons, T.; SHiLS, E. (1951). Toward a General Theory of Action. Cambridge, Mass.: Harvard Universiry Press.

Ritzert, G. (1992), (1993). "Teoría de redes". Teoria Sociologica Contemporánea. Madrid, [Etc]: McGraw-Hill, p. 447-450, 562- 564.

ROSE, M. (1975). Industrial Behaviour. Harmondsworth: Allen Lane.

Scort, John (1991). Social Network Analysis. Newbury Park, Londres: Sage.

SNYDER, D.; KICK, E.L. (1979). "Structural position in the world system an economic growth, 1975-1970: a multiple nenwork analysis of transnational interactions". En American Journal of Sociology 84: 1096-1126.

SONQUIST, J.A.; KOENIG, T. (1975). "Interlocking directorates in the top US corporations: a graph theory approach». En lnsurgent Sociology 5: 196-229.

STRUCTURE. Versión 4.2. (1991). Programa de ordenador. Nueva York: Center for Social Sciences. Columbia University

TiCHY, N.; FOMBRUN, CH. (1979). "Network analysis in organizational settings". En Human Relations 32: 923- 965.

VAN DEL POEL, Mart GM. (1993). "Delineating personal support networks". En Social Networks 15: 49-70.

WASSERMAN, S; FAUST, K. (1994). Social Network Aanalysis. Cambridge: University Press.

WallaCE, W.L. (ed.) (1969). Sociological Theory. An Introduction. Chicago: Aldine.

WeBER, M. (1947). "The Theory of Social and Economic Organization". Nueva York: Free Press.

Wellman, B. (1983). "Network Analysis: Some Basic Principles". En Collins, R. Sociological Theory-1983. San Francisco: Jossey-Bass, p. 155-200.

WELIMAN, B.; BERKOWITZ, S.D. (1991). "Introduction: Studying social structure". En Weilman, B.; Berkowitz, S.D., (ed.). Social Structure. A Network Approach. Cambridge, MA: Cambridge University Press.

- (ed.) (1991). Social Structure. A Network Approach. Cambridge MA.: Cambridge University Press.

WHITE, H. (1963). An Anatomy of Kinship. Englewood Cliffs, NJ: Prentice-Hall.

WHITE, H.; BOORMAN, S.A.; BREIGER, R.L. (1976). "Social Structure from Multiple Networks I. Blockmodels of Roles and Positions". En American Journal of Sociology 81: 730-779.

WINSHIP, C.; MANDEL, M. (1984). "Roles and Positions: A critique and Extension of the Blockmodelling Approachw. En Leinhardt, S. (ed.). Social Networks: A Developing Paradigm. Nueva York: Academic Press. 